11

\title{
Анализ поглощающих периодических структур, образованных цилиндрическими электромагнитными черными дырами
}

\author{
(C) Я.И. Чижевская, ${ }^{1}$ О.Н. Смольникова, ${ }^{2,3}$ С.П. Скобелев ${ }^{1,3,9}$ \\ ${ }^{1}$ Московский физико-технический институт (национальный исследовательский университет), \\ 141701 Долгопрудный, Московская обл., Россия \\ 2 Московский авиационный институт (национальный исследовательский университет), \\ 125080 Москва, Россия \\ ${ }^{3}$ Публичное акционерное общество „Радиофизика», \\ 125363 Москва, Россия \\ ฯe-mail: s.p.skobelev@mail.ru
}

Поступило в Редакцию 3 июня 2020 г.

В окончательной редакции 9 июля 2020 г.

Принято к публикации 16 июля 2020 г.

Рассмотрена двумерная задача рассеяния плоской электромагнитной волны на одномерно-периодических структурах с цилиндрическими элементами типа электромагнитных черных дыр, расположенных на полубесконечной подложке. Разработаны два алгоритма решения, соответствующих случаям $E$ - и $H$-поляризации и основанных на гибридном проекционном методе с учетом распределения профилей показателя преломления в черных дырах различного типа. Алгоритмы обобщены также и на случай расположения черных дыр на идеально проводящем экране. Приведен ряд численных результатов, характеризующих как эффективность самих алгоритмов, так и характеристики поглощающих структур с черными дырами. Проведено сравнение характеристик поглощения в указанных структурах с аналогичными характеристиками для структур с клиновидными элементами.

Ключевые слова: неоднородные среды, периодические структуры, поглощение волн, рассеяние волн, численные методы, электромагнитные черные дыры.

DOI: 10.21883/JTF.2021.02.50369.191-20

\section{Введение}

Исследования и разработки поглощающих структур в СВЧ и оптическом диапазонах представляют большой интерес в течение долгого времени, так как они находят ряд важных практических приложений, включая безэховые камеры для измерения характеристик антенн и СВЧ-устройств, средства электромагнитной совместимости, покрытия для снижения ЭПР радиолокационных объектов и солнечные батареи.

Современные требования к поглощающим структурам включают почти 100\% эффективность поглощения в широкой полосе частот и в широком секторе углов облучения. Один из перспективных типов поглощающих элементов, отвечающий требованиям, указанными выше, - так называемая оптическая (или электромагнитная) черная дыра, предложенная в [1]. Поглотитель в [1] состоит из однородной цилиндрической центральной части с комплексной диэлектрической проницаемостью и неоднородной оболочки без потерь с относительной проницаемостью $\varepsilon(\rho)=(a / \rho)^{2}$, где $a-$ внешний радиус и $\rho-$ радиальная координата. Последующие исследования указанного поглотителя были проведены в [2-5].

Модифицированная версия поглотителя [1], в которой в отличие от [1] непрерывность проницаемости обеспечивается не только на внешней границе поглотителя, но и на границе между оболочкой и центральной частью, предложена и исследована в [6]. Работа [6] также включает в рассмотрение поглощающие свойства цилиндрической линзы Люнеберга-Гутмана [7] с потерями.

Еще одна версия, которую ее авторы называют фотонной черной дырой, с проницаемостью $\varepsilon(\rho)=(a / \rho)^{v}$ в общем случае была исследована в [8], где, в частности, была рассмотрена фотонная ловушка, соответствующая $v>2$.

Наконец, авторы статьи [9] предложили радиальнонеоднородный поглотитель, названный метаматериальной черной дырой, которая в отличие от предыдущих чисто диэлектрических модификаций (с относительной магнитной проницаемостью $\mu=1)$ использует оболочку с диэлектрической и магнитной проницаемостями, заданными в виде $\varepsilon(\rho)=\mu(\rho)=\alpha a^{2} / \rho^{2}$, где $\alpha-$ комплексный параметр с действительной частью, равной -1 . Как хорошо известно, среды с одновременно отрицательными $\varepsilon$ и $\mu$ обладают рядом замечательных свойств, описанных в [10]. Согласно [9], применение такого материала в предположении очень маленьких величин потерь у внешней поверхности и очень маленького радиуса центральной области теоретически позволяет получать эффективное поперечное сечение поглощения, многократно превышающее классический предел для эффективного сечения поглощения для черных тел большого размера. Недавние исследования характеристик цилиндрических черных дыр с профилем проницаемостей, 
указанных в [9], с реалистичными параметрами потерь у поверхности и радиуса центральной части проведены в [11].

Обзор публикаций, указанных выше, показывает, что одиночные поглотители в виде черных дыр различных модификаций уже достаточно подробно исследованы. Однако работ, где бы рассматривались черные дыры в виде элементов поглощающих периодических структур, найдено не было, хотя примеры структур с цилиндрическими и сферическими поглощающими элементами других типов имеются [12-14].

Цель настоящей работы - сравнительный анализ характеристик рассеяния и поглощения электромагнитных волн одномерно-периодическими структурами, элементами которых являются черные дыры нескольких модификаций, в строгой постановке электродинамической задачи. Кроме того, результаты, полученные для структур с черными дырами, будут сопоставлены с аналогичными результатами, соответствующими структурам с известными клиновидными поглощающими элементами.

\section{1. Постановка задачи}

Сечение базового варианта одномерно-периодической структуры, которая будет анализироваться в настоящей работе, показано на рис. 1 в прямоугольной декартовой системе координат $x, y$ и $z$. Структура образована двуслойными круговыми цилиндрическими элементами внешнего радиуса $a$, расположенными с периодом $b$ на подложке полубесконечной толщины. Внешний слой каждого элемента считается неоднородным с относительной диэлектрической проницаемостью $\varepsilon(x, z)$ и относительной магнитной проницаемостью $\mu(x, z)$, зависящими от радиальной координаты $\rho=\left[x^{2}+(z-a)^{2}\right]^{1 / 2}$, отмеряемой от оси цилиндра. Центральная область элемента представляет собой однородный цилиндр радиуса $a_{1}$ с относительными постоянными проницаемостями $\varepsilon_{1}$ и $\mu_{1}$. Конкретные профили проницаемостей элемента будут рассмотрены в разд. 3. Подложка считается однородным полупространством с относительными проницаемостями $\varepsilon_{s}$ и $\mu_{s}$. Проницаемости всего остального пространства вне цилиндров считаются равными единице. Частный случай $\varepsilon_{s}=\mu_{s}=1$ соответствует

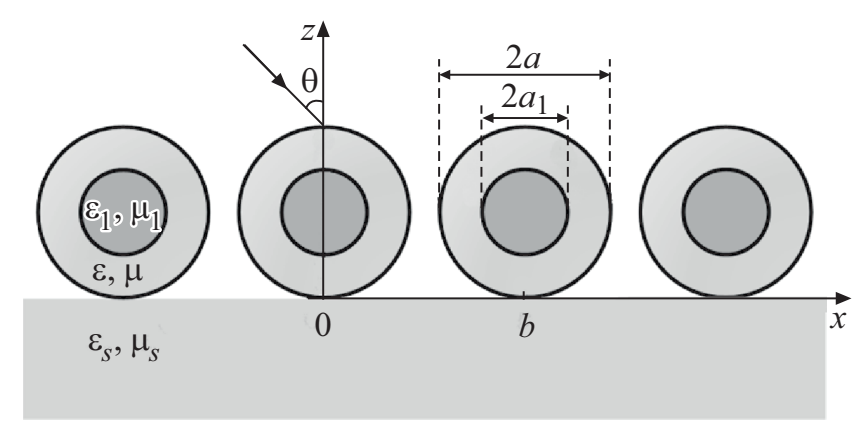

Рис. 1. Геометрия задачи. симметричной конфигурации, представляющей интерес для некоторых приложений [12]. Еще одним частным случаем, который мы также рассмотрим ниже, является наличие идеально проводящего экрана, расположенного в плоскости $z=0$.

Предполагается, что структура возбуждается электромагнитной плоской волной единичной амплитуды с гармонической зависимостью от времени $\exp (-i \omega t)$, распространяющейся под углом $\theta$ к оси $z$ (рис. 1), и требуется определить поле, рассеянное структурой, а также мощность, поглощенную элементами структуры. Ниже мы рассмотрим случай $E$-поляризации, соответствующий горизонтальной поляризации падающей волны, и случай $H$-поляризации, соответствующий падающей волне, поляризованной в вертикальной плоскости $0 x z$.

\section{2. Метод решения}

Задача, поставленная выше, может быть решена несколькими численными методами, включая метод интегральных уравнений для токов поляризации $[15,16]$, метод конечных элементов [17], метод конечных разностей во временной области [18], дифференциальный метод [19] и гибридный проекционный метод [20]. Обзоры по методам [15-18] имеются в [21] и частично в [20]. Мы будем решать задачу с использованием гибридного проекционного метода [20]. Указанный метод основан на подходе [22], где проектирование уравнений Гельмгольца для полей сочетается с применением одномерного метода конечных элементов в проекционной форме к решению полученных систем обыкновенных дифференциальных уравнений. Такой подход затем был развит для анализа волноводных решеток с выступающими однородными диэлектрическими элементами [23] и чисто диэлектрических согласующих и поглощающих структур [20]. Здесь мы обобщаем его на структуры, элементы которых, как указано выше, являются неоднородными как по диэлектрической проницаемости, так и по магнитной.

\section{1. $E$-поляризация}

Составляющие векторов напряженности поля, не равные нулю тождественно в рассматриваемом случае, включают составляющую напряженности электрического поля $E_{y}$, а также составляющие напряженности магнитного поля $H_{x}$ и $H_{z}$, определяемые через $E_{y}$ из уравнений Максвелла. Следуя [20], представим полное электрическое поле в свободном пространстве над цилиндрами $(z \geq h, h=2 a)$ и в подложке $(z \leq 0)$ в виде разложений по гармоникам Флоке

$$
\begin{aligned}
E_{y}(x, z \geq h)= & \sum_{q=-\infty}^{\infty}\left\{\delta_{q 0} \exp \left[-i \Gamma_{q}(z-h)\right]\right. \\
& \left.+R_{q} \exp \left[i \Gamma_{q}(z-h)\right]\right\} \exp \left(i \alpha_{q} x\right),
\end{aligned}
$$




$$
E_{y}(x, z \leq 0)=\sum_{q=-\infty}^{\infty} T_{q} \exp \left(i \alpha_{q} x-i \Gamma_{s q} z\right),
$$

где первое слагаемое в (1) соответствует падающей волне, $\delta_{q 0}-$ символ Кронекера, $R_{q}$ и $T_{q}-$ неизвестные амплитуды отраженных волн и волн, прошедших в подложку, $\alpha_{q}=k \sin \theta+2 \pi q / b-$ поперечные постоянные распространения,

$$
\Gamma_{q}=\sqrt{k^{2}-\alpha_{q}^{2}}, \quad \Gamma_{s q}=\sqrt{k^{2} \varepsilon_{s} \mu_{s}-\alpha_{q}^{2}}
$$

- продольные постоянные распространения, в свободном пространстве и подложке соответственно, $k=2 \pi / \lambda-$ волновое число и $\lambda-$ длина волны в свободном пространстве.

Напряженность электрического поля в области $0 \leq z \leq h$, содержащей цилиндры, представляется в виде разложения по полной системе поперечных функций гармоник Флоке

$$
E_{y}(x, 0 \leq z \leq h)=\sum_{q=-\infty}^{\infty} E_{q}(z) \exp \left(i \alpha_{q} x\right)
$$

с неизвестными переменными коэффициентами $E_{q}(z)$.

Электрические поля (1), (2) и (4), а также соответствующие поперечные магнитные поля, определяемые из уравнений Максвелла формулой

$$
H_{x}=-\frac{1}{i \omega \mu_{0} \mu} \frac{\partial E_{y}}{\partial z},
$$

где $\mu_{0}-$ магнитная постоянная для свободного пространства и $\mu$ - распределение относительной магнитной проницаемости в соответствующей области, должны быть непрерывными на границах областей при $z=0$ и $z=h$. Выполнение указанного условия и ортогональность поперечных функций $\exp \left(-i \alpha_{p} x\right)$ приводят к следующим соотношениям между коэффициентами разложения:

$$
\begin{gathered}
R_{q}=E_{q}(h)-\delta_{q 0} \\
\sum_{q=-\infty}^{\infty} \tilde{\mu}_{p q}(h) E_{q}^{\prime}(h)=\left[E_{p}(h)-2 \delta_{p 0}\right] i \Gamma_{p} \\
T_{q}=E_{q}(0) \\
\sum_{q=-\infty}^{\infty} \tilde{\mu}_{p q}(0) E_{q}^{\prime}(0)=-\frac{i \Gamma_{s p}}{\mu_{s}} E_{p}(0)
\end{gathered}
$$

где штрих у $E_{q}$ означает производную по аргументу,

$$
\tilde{\mu}_{p q}(z)=\frac{1}{b} \int_{-b / 2}^{b / 2} \frac{\exp \left[i\left(\alpha_{q}-\alpha_{p}\right) x\right]}{\hat{\mu}(x, z)} d x
$$

$\hat{\mu}(x, z)=1$ вне цилиндра, $\hat{\mu}(x, z)=\mu(x, z)$ во внешнем слое элемента и $\hat{\mu}(x, z)=\mu_{1}$ в центральной области.
Поле (4) в неоднородной области должно удовлетворять уравнению Гельмгольца

$$
\frac{\partial}{\partial z}\left(\frac{1}{\hat{\mu}} \frac{\partial E_{y}}{\partial z}\right)+\frac{\partial}{\partial x}\left(\frac{1}{\hat{\mu}} \frac{\partial E_{y}}{\partial x}\right)+k^{2} \hat{\varepsilon} E_{y}=0,
$$

следующему из уравнений Максвелла, где $\hat{\varepsilon}(x, z)=1$ вне цилиндра, $\hat{\varepsilon}(x, z)=\varepsilon(x, z)$ во внешнем слое элемента и $\hat{\varepsilon}(x, z)=\varepsilon_{1}$ в центральной области. Проектирование (11) на поперечные функции и учет разложения (4) приводит к системе обыкновенных дифференциальных уравнений

$$
\begin{aligned}
\frac{d}{d Z} \sum_{q=-\infty}^{\infty} \tilde{\mu}_{p q}(z) E_{q}^{\prime}(z) & -\sum_{q=-\infty}^{\infty} \alpha_{p} \alpha_{q} \tilde{\mu}_{p q}(z) E_{q}(z) \\
& +k^{2} \sum_{q=-\infty}^{\infty} \varepsilon_{p q}(z) E_{q}(z)=0
\end{aligned}
$$

где

$$
\varepsilon_{p q}(z)=\frac{1}{b} \int_{-b / 2}^{b / 2} \hat{\varepsilon}(x, z) \exp \left[i\left(\alpha_{q}-\alpha_{p}\right) x\right] d x
$$

и $p=0, \pm 1, \pm 2, \ldots$.

Система уравнений (12) решается далее с использованием одномерного метода конечных элементов. Неизвестные переменные коэффициенты разложения в (4) представляются в виде разложения

$$
E_{q}(z)=\sum_{n=1}^{N} E_{n q} f_{n}(z)
$$

где $E_{n q}-$ неизвестные, но уже постоянные, коэффициенты и $f_{n}(r)$ треугольные функции, например, [20], с вершинами, расположенными в $N$ точках с координатами $z_{n}=(n-1) \Delta, \Delta=h /(N-1)$. Теперь мы подставляем (14) в (12), умножаем (12) на $f_{m}(z)$ и интегрируем произведение по $z$ от 0 до $h$. Интегрирование первого слагаемого произведения по частям приводит к двум дополнительным слагаемым вне интеграла, которые мы заменяем согласно (7) и (9). В результате мы сводим (12) к системе линейных алгебраических уравнений

$$
\begin{aligned}
\frac{i \Gamma_{s p}}{\mu_{s} k} E_{1 p} \delta_{m 1} & +\sum_{n=1}^{N} \sum_{q=-\infty}^{\infty} Z_{m n}^{p q} E_{n q}+\frac{i \Gamma_{p}}{k} E_{N p} \delta_{m N} \\
& =\frac{2 i \Gamma_{p}}{k} \delta_{p 0} \delta_{m N}
\end{aligned}
$$

где

$$
Z_{m n}^{p q}=I_{1 m n}^{p q}-\frac{\alpha_{p} \alpha_{q}}{k^{2}} I_{2 m n}^{p q}-I_{3 m n}^{p q}
$$

- матричные элементы, определяемые интегралами

$$
I_{1 m n}^{p q}=k \int_{0}^{h} f_{m} f_{n} \varepsilon_{p q} d z
$$




$$
\begin{aligned}
& I_{2 m n}^{p q}=k \int_{0}^{h} f_{m} f_{n} \tilde{\mu}_{p q} d z, \\
& I_{3 m n}^{p q}=k \int_{0}^{h} f_{m}^{\prime} f_{n}^{\prime} \tilde{\mu}_{p q} d z .
\end{aligned}
$$

$E_{1 p}=E_{p}(0), E_{N p}=E_{p}(h)$ и $m=1,2, \ldots, N$.

Бесконечная система (15) решается методом усечения с удержанием $P$ первых членов в разложениях (1), (2) и (4), соответствующих $q=0, \pm 1, \ldots, \pm Q$ так что $P=1+2 Q$. Порядок усеченной системы оказывается равным $P \times N$, а матрица системы имеет блочную трехдиагональную структуру [20]. Численное решение полученной системы позволяет далее рассчитать амплитуды отраженных и прошедших волн согласно формулам (6) и (8) соответственно.

\section{2.}

В случае $H$-поляризации основная ненулевая составляющая электромагнитного поля $H_{y}$ в трех областях, рассматриваемых в задаче, представляется разложениями, аналогичными (1), (2) и (4):

$$
\begin{array}{r}
H_{y}(x, z \geq h)=\sum_{q=-\infty}^{\infty}\left\{\delta_{q 0} \exp \left[-i \Gamma_{q}(z-h)\right]\right. \\
\left.+R_{q} \exp \left[i \Gamma_{q}(z-h)\right]\right\} \exp \left(i \alpha_{q} x\right), \\
H_{y}(x, z \leq 0)=\sum_{q=-\infty}^{\infty} T_{q} \exp \left(i \alpha_{q} x-i \Gamma_{s q} z\right), \\
H_{y}(x, 0 \leq z \leq h)=\sum_{q=-\infty}^{\infty} H_{q}(z) \exp \left(i \alpha_{q} x\right) .
\end{array}
$$

Соответствующие поперечные составляющие напряженности электрического поля, которые потребуются в последующих операциях, определяются формулой

$$
E_{x}=\frac{1}{i \omega \varepsilon_{0} \varepsilon} \frac{\partial H_{y}}{\partial z}
$$

где $\varepsilon_{0}-$ электрическая постоянная для свободного пространства и $\varepsilon$ - распределение относительной диэлектрической проницаемости в соответствующей области.

Проекционное сшивание поперечных магнитных и электрических полей на границах областей приводит к соотношениям

$$
\begin{gathered}
R_{q}=H_{q}(h)-\delta_{q 0}, \\
\sum_{q=-\infty}^{\infty} \tilde{\varepsilon}_{p q}(h) H_{q}^{\prime}(h)=\left[H_{p}(h)-2 \delta_{p 0}\right] i \Gamma_{p}, \\
T_{q}=H_{q}(0), \\
\sum_{q=-\infty}^{\infty} \tilde{\varepsilon}_{p q}(0) H_{q}^{\prime}(0)=-\frac{i \Gamma_{s p}}{\varepsilon_{s}} H_{p}(0),
\end{gathered}
$$

где

$$
\tilde{\varepsilon}_{p q}(z)=\frac{1}{b} \int_{-b / 2}^{b / 2} \frac{\exp \left[i\left(\alpha_{q}-\alpha_{p}\right) x\right]}{\hat{\varepsilon}(x, a)} d x .
$$

Магнитное поле (22) должно удовлетворять уравнению Гельмгольца

$$
\frac{\partial}{\partial z}\left(\frac{1}{\hat{\varepsilon}} \frac{\partial H_{y}}{\partial z}\right)+\frac{\partial}{\partial x}\left(\frac{1}{\hat{\varepsilon}} \frac{\partial H_{y}}{\partial x}\right)+k^{2} \hat{\mu} H_{y}=0 .
$$

Подставляя (22) в (29) и проектируя (29) на поперечные функции, мы получаем систему обыкновенных дифференциальных уравнений

$$
\begin{aligned}
& \frac{d}{d z} \sum_{q=-\infty}^{\infty} \tilde{\varepsilon}_{p q}(z) H_{q}^{\prime}(z)-\sum_{q=-\infty}^{\infty} \alpha_{p} \alpha_{q} \tilde{\varepsilon}_{p q}(z) H_{q}(z) \\
& \quad+k^{2} \sum_{q=-\infty}^{\infty} \mu_{p q}(z) H_{q}(z)=0,
\end{aligned}
$$

где

$$
\mu_{p q}(z)=\frac{1}{b} \int_{-b / 2}^{b / 2} \hat{\mu}(x, z) \exp \left[i\left(\alpha_{q}-\alpha_{p}\right) x\right] d x
$$

Представляя $H_{q}(z)$ в виде разложения

$$
H_{q}(z)=\sum_{n=1}^{N} H_{n q} f_{n}(z)
$$

и применяя метод конечных элементов к решению системы (30), как описано выше, мы сводим задачу к системе линейных алгебраических уравнений для постоянных коэффициентов $H_{n q}$

$$
\begin{gathered}
\frac{i \Gamma_{s p}}{\varepsilon_{s} k} H_{1 p} \delta_{m 1}+\sum_{n=1}^{N} \sum_{q=-\infty}^{\infty} Z_{m n}^{p q} H_{n q}+\frac{i \Gamma_{p}}{k} H_{N p} \delta_{m N} \\
=\frac{2 i \Gamma_{p}}{k} \delta_{p 0} \delta_{m N} .
\end{gathered}
$$

где $m=1,2, \ldots, N$. Матричные элементы в (33) также определяются формулой (16), но $\varepsilon_{p q}$ в (17) должно быть заменено на $\mu_{p q}$, а в (18) и (19) должно быть заменено на $\tilde{\varepsilon}_{p q}$. Решив систему (33) методом усечения, как описано выше, мы можем определить амплитуды рассеянных волн, стоящие в (20) и (21), по формулам (24) и (26), где $H_{p}(0)=H_{1 p}$ и $H_{p}(h)=H_{N p}$.

\section{3. Случай структуры на экране}

Если элементы структуры помещены на идеально проводящий экран, расположенный в плоскости $z=0$, то алгоритмы, описанные выше, могут быть модифицированы следующим образом. Во-первых, ясно, что поля 
(2) и (21) исключаются из рассмотрения. Во-вторых, условие равенства нулю поперечного электрического поля на экране приводит к соотношению

$$
E_{q}(0)=0
$$

вместо (8) и (9) в случае $E$-поляризации, и к соотношению

$$
\sum_{q=-\infty}^{\infty} \tilde{\varepsilon}_{p q}(0) H_{q}^{\prime}(0)=0
$$

вместо (26) и (27) в случае $H$-поляризации.

Условие (34) означает, что первая треугольная функция в (14) должна быть равной нулю при $z=0$, и, следовательно, вершины треугольных функций теперь должны располагаться в точках с координатами $z_{n}=n \Delta$, где $\Delta=h / N$. Тот факт, что $f 1(0)=0$, также исключает первое слагаемое из уравнений (15) для случая $E$ поляризации.

Первое слагаемое, стоящее в уравнениях (33) для случая $H$-поляризации, также исключается из указанных уравнений, но уже благодаря соотношению (35). Однако координаты вершин треугольных функций в разложении (32) остаются таким же, как и в отсутствии экрана.

\section{3. Реализация алгоритмов и численные результаты}

Алгоритмы, описанные выше, были реализованы в нескольких компьютерных программах на языке MATLAB. Интегралы типа (13) вычислялись с помощью метода Филона. Интегралы (17)-(19) рассчитывались с использованием кусочно-линейной аппроксимации функций (13) и аналогичных функций от $z$, как это делается в (14) и (32). Контроль точности расчетов осуществлялся с помощью соотношения энергетического баланса

$$
P^{R}+P^{T}+P_{\varepsilon}^{A}+P_{\mu}^{A}=P^{I}
$$

где $P^{I}=\cos \theta \quad-\quad$ падающая мощность, $P^{R}=\sum_{q}\left|R_{q}\right|^{2} \operatorname{Re}\left(\Gamma_{q} / k\right)$ - отраженная мощность, $P^{T}=\frac{1}{\mu_{s}} \sum_{q}\left|T_{q}\right|^{2} \operatorname{Re}\left(\Gamma_{s q} / k\right)$ - прошедшая мощность и

$$
\begin{gathered}
P_{\varepsilon}^{A}=\sum_{m=1}^{N} \sum_{n=1}^{N} \sum_{p, q} E_{m p}^{*} E_{n q} \operatorname{Im}\left\{I_{1 m n}^{p q}\right\}, \\
P_{\mu}^{A}=-\sum_{m=1}^{N} \sum_{n=1}^{N} \sum_{p, q} E_{m p}^{*} E_{n q} \times \operatorname{Im}\left(\alpha_{p} \alpha_{q} I_{2 m n}^{p q} / k^{2}+I_{3 m n}^{p q}\right)
\end{gathered}
$$

- мощности, поглощенные на периоде структуры из-за диэлектрических и магнитных потерь. Выражения, приведенные выше, соответствуют случаю $E$-поляризации и нормированы на $k b /\left(2 \omega \mu_{0}\right)$. Соотношения для случая $H$-поляризации имеют аналогичную форму и поэтому не приводятся. Так как соотношения типа (36) являются только необходимым условием для правильного решения задачи, то следует проводить также и другие
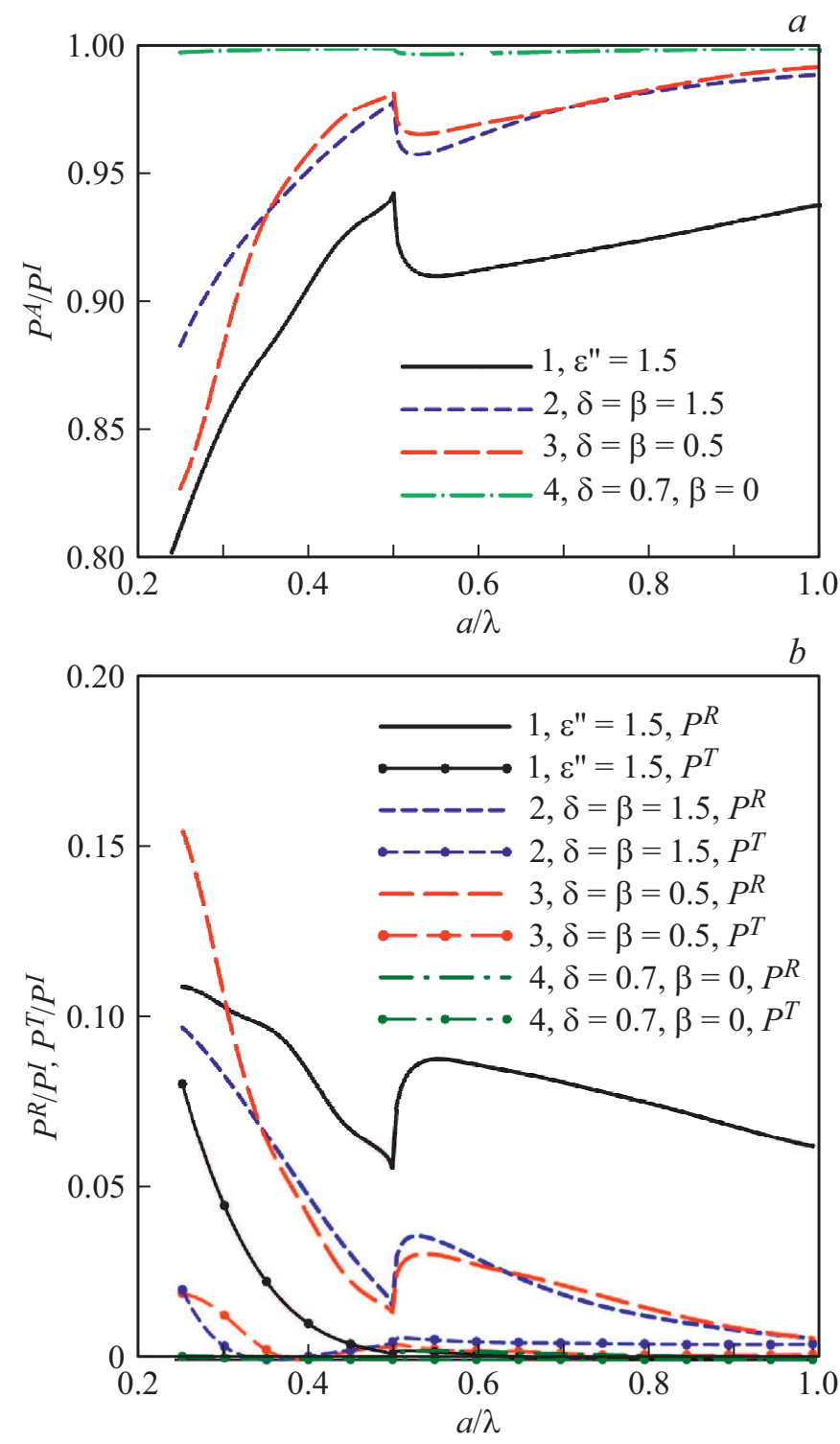

Рис. 2. Поглощенная $(a)$, а также отраженная и прошедшая $(b)$ мощности для структуры с $a_{1} / a=0.5$ в свободном пространстве $\left(\varepsilon_{s}=m_{s}=1\right)$ при нормальном падении $(\theta=0)$ волны; $E$-поляризация

проверки работы алгоритмов, включая исследование сходимости результатов и их совпадение с результатами, полученными другими способами для тех или иных структур. Такие достаточно подробные исследования метода были проведены в наших предыдущих работах [20] и [23], откуда следуют рекомендации по его применению, которыми мы и пользовались при расчете характеристик рассматриваемых структур.

Разработанные алгоритмы были использованы для численного исследования следующих поглотителей:

1. Поглотитель [1] с оболочкой без потерь с проницаемостью $\varepsilon(\rho)=(a / \rho)^{2}$ и $\varepsilon_{1}=\varepsilon\left(a_{1}\right)+i \varepsilon^{\prime \prime}$ в центральной части, $\mu=\mu_{1}=1$. 

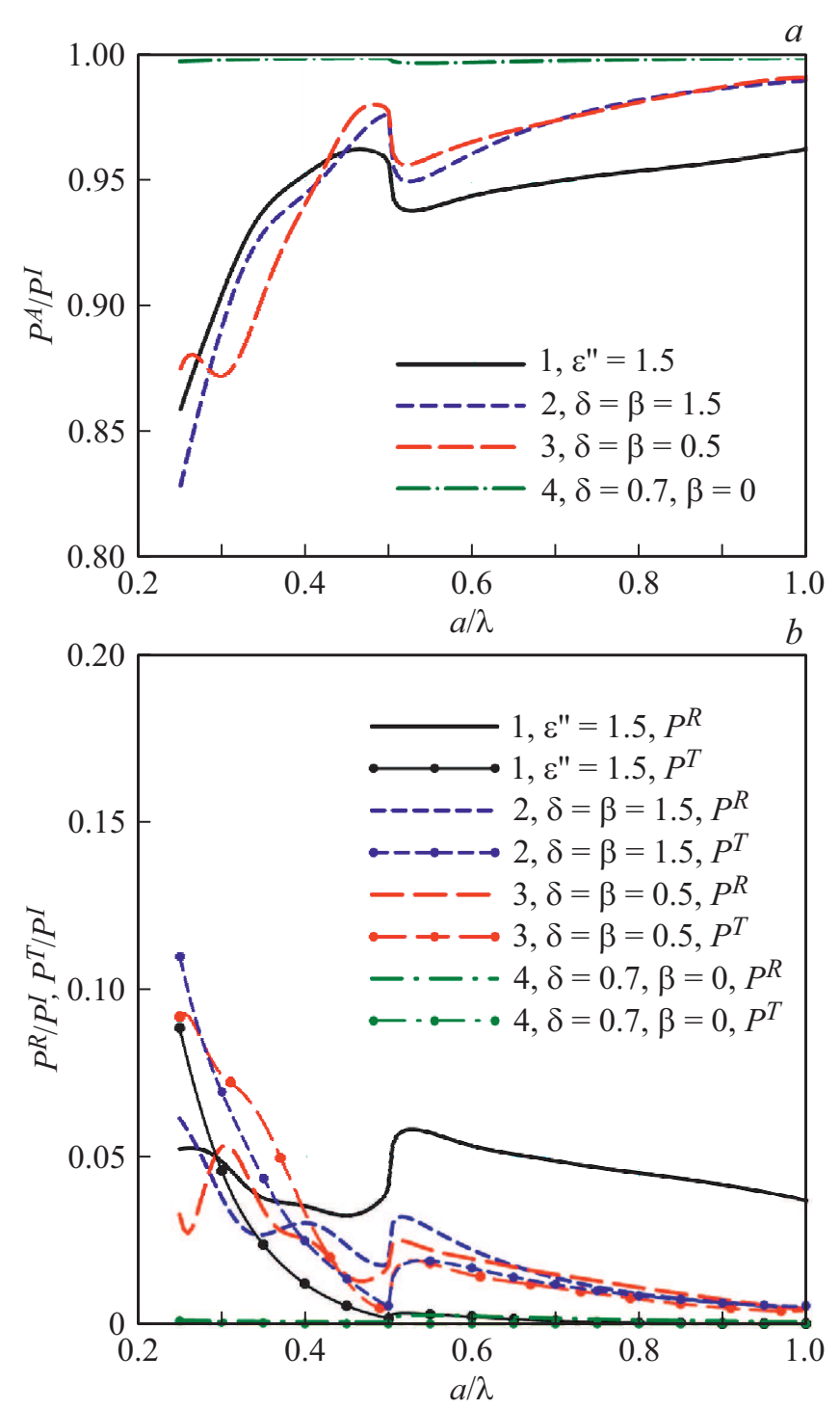

Рис. 3. То же, что на рис. 2, но для $H$-поляризации.

2. Модификация поглотителя [6], обеспечивающая непрерывность комплексной диэлектрической проницаемости на внутренней границе цилиндров с диэлектрической проницаемостью в оболочке $\varepsilon(\rho)=(1+i \delta)(a / \rho)^{2}-i \beta \quad$ и $\quad \varepsilon_{1}=\varepsilon\left(a_{1}\right) \quad \mu=\mu=1 \quad$ в центре, $\mu=\mu_{1}=1$.

3. Поглотитель типа [8] с проницаемостью в оболочке $\varepsilon(\rho)=(1+i \delta)(a / \rho)^{4}-i \beta$ и $\varepsilon_{1}=\varepsilon\left(a_{1}\right), \mu=\mu_{1}=1$.

4. Поглотитель типа [9] с $\varepsilon(\rho)=\mu(\rho)=(1+i \delta) \times$ $\times(a / \rho)^{2}-i \beta$ в оболочке и $\varepsilon_{1}=\mu_{1}=\varepsilon\left(a_{1}\right)$ в центральной области.

Параметр $\delta$ в характеристиках поглотителей 2-4 позволяет варьировать уровень потерь. Параметр $\beta=0$ соответствует случаю, когда поверхность поглотителей 2 и 3 не согласована со свободным пространством по мнимой части, а при $\beta=\delta$ внешняя поверхность оказывается полностью согласованной.
Численные эксперименты показали следующие результаты. Во-первых, наивысшая эффективность поглощения, как и ожидалось, достигается при расположении элементов вплотную друг к другу, т.е. при $b=2 a$. Указанное расположение далее использовалось при последующей оптимизации параметров $\varepsilon^{\prime \prime}, a_{1} / a, \delta$ и $\beta$ для каждого типа поглотителя с целью получения максимальной эффективности поглощения. Расчеты показали, что хорошие результаты получаются, когда $a_{1} / a$ находится в диапазоне от 0.3 до 0.5 для всех типов, тогда как оптимальные значения $\delta$ и $\beta$ сильнее зависят от типа поглотителя. Наилучшие результаты для поглотителей 2 и 3 достигаются при согласовании их внешних поверхностей, т. е. при $\beta=\delta$. Поверхность поглотителя 4 не требует дополнительного согласования, так как импеданс среды поглотителя всегда равен импедансу свободного пространства, и поэтому использовалось значение $\beta=0$. Численные значения параметров указаны на рисунках, описываемых ниже.
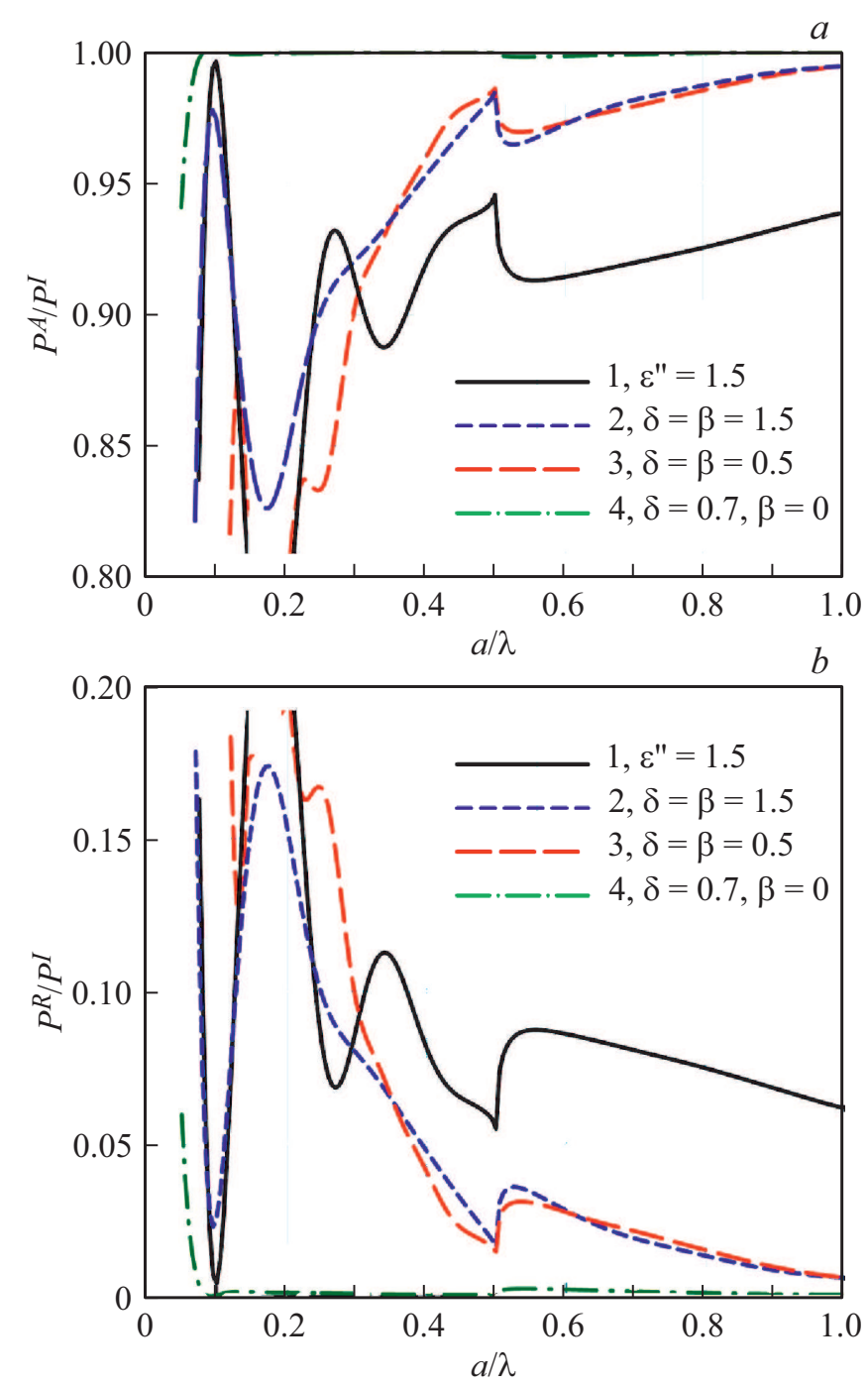

Рис. 4. Поглощенная $(a)$, а также отраженная и прошедшая $(b)$ мощности для структуры с $a_{1} / a=0.5$ на экране при нормальном падении $(\theta=0)$ волны; $E$-поляризация. 

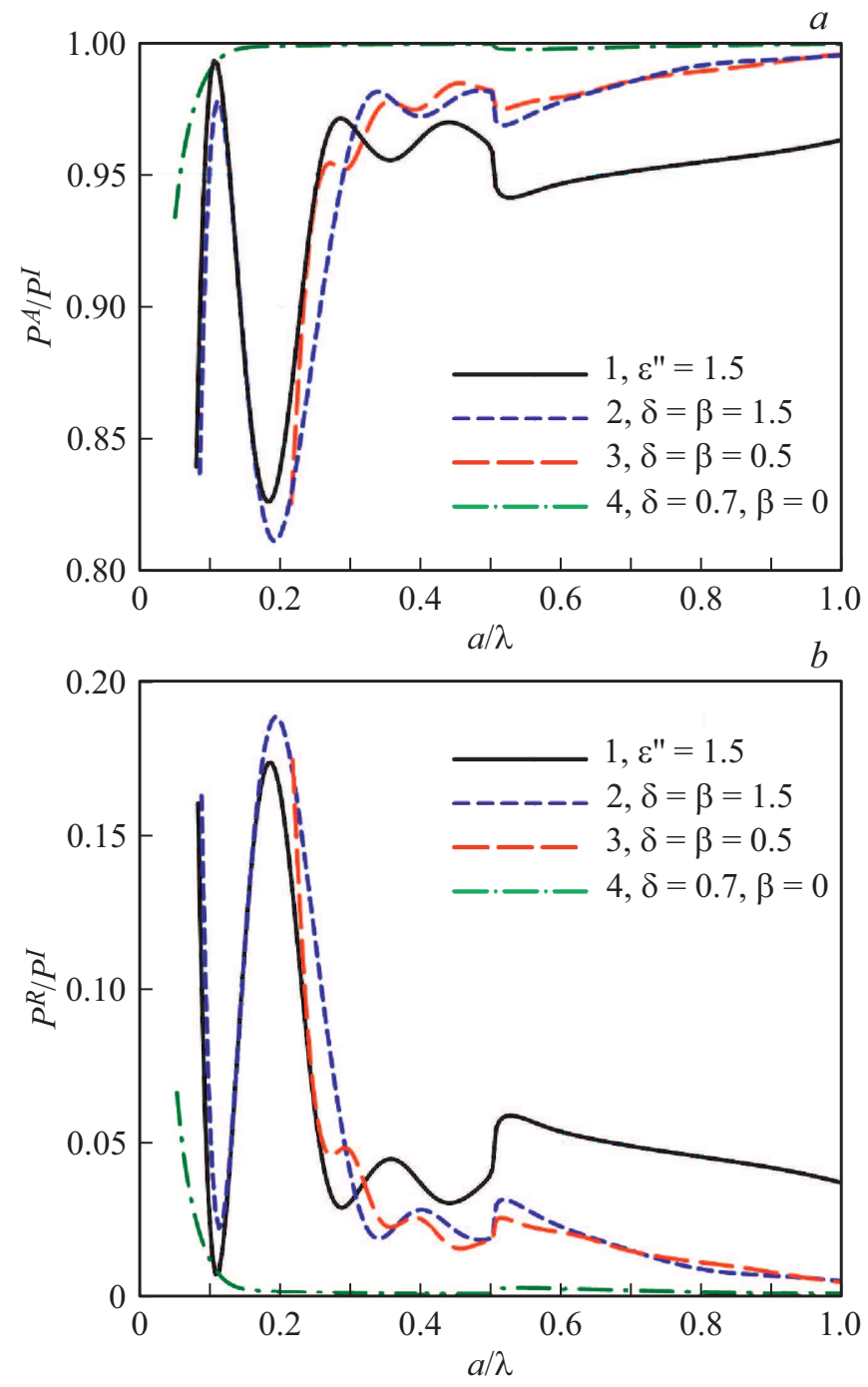

Pис. 5. То же, что на рис. 4 , но для $H$-поляризации.

Результаты, соответствующие параметрам $a_{1} / a=0.5$ и $\varepsilon_{s}=m_{s}=1$ (как в [12]), при нормальном падении плоской волны $(\theta=0)$ в зависимости от радиуса а приведены на рис. 2 и 3 для случаев $E$ - и $H$-поляризации соответственно. Результаты для поглощенной мощности, нормированной на падающую мощность как функции радиуса цилиндра, представлены на рис. 2, a и 3, $a$. Можно заметить, что поглотитель 4 имеет наиболее высокую эффективность поглощения, начиная уже с $a=0.25 \lambda$. Поглощенная мощность для элементов других типов стремится к значениям поглощенной мощности 4 при увеличении радиуса $a$. Поглотители 2 и 3 обладают близкими друг к другу характеристиками, хотя оптимальные значения параметра $\delta$ у них различны. Рис. $2, b$ и $3, b$ содержат кривые для отраженной и прошедшей мощностей, нормированных на падающую мощность, для случаев $E$ - и $H$-поляризации соответственно при $a 1 / a=0.5$. Как мы видим, уровень прошедшей мощности заметно ниже, чем уровень отраженной.
Приведенные кривые проявляют аномальное поведение вблизи $a=0.5 \lambda$, которое объясняется тем что - 1-я и 1-я пространственные гармоники, являющиеся затухающими при $a<0.5 \lambda$, становятся распространяющимися при $a>0.5 \lambda$. Аномалии, также имеющие место вблизи значений $a=\lambda, 1.5 \lambda, \ldots$, уже выражены в меньшей степени.

Результаты, полученные для поглощенной и отраженной мощностей в случае структуры с параметрами элементов, указанными выше, но расположенной на идеально проводящем экране, приведены на рис. 4 и 5 для $E$ - и $H$-поляризации соответственно. Как мы видим, наличие экрана заметно проявляется лишь при малых значениях внешнего радиуса поглотителя. Благодаря повышению эффективности поглощения при увеличении радиуса цилиндра, наличие подложки с $\varepsilon_{s}>1$ и $\mu_{s}>1$ дает результаты, слабо отличающиеся от результатов, указанных выше, и поэтому они не приводятся здесь для краткости.
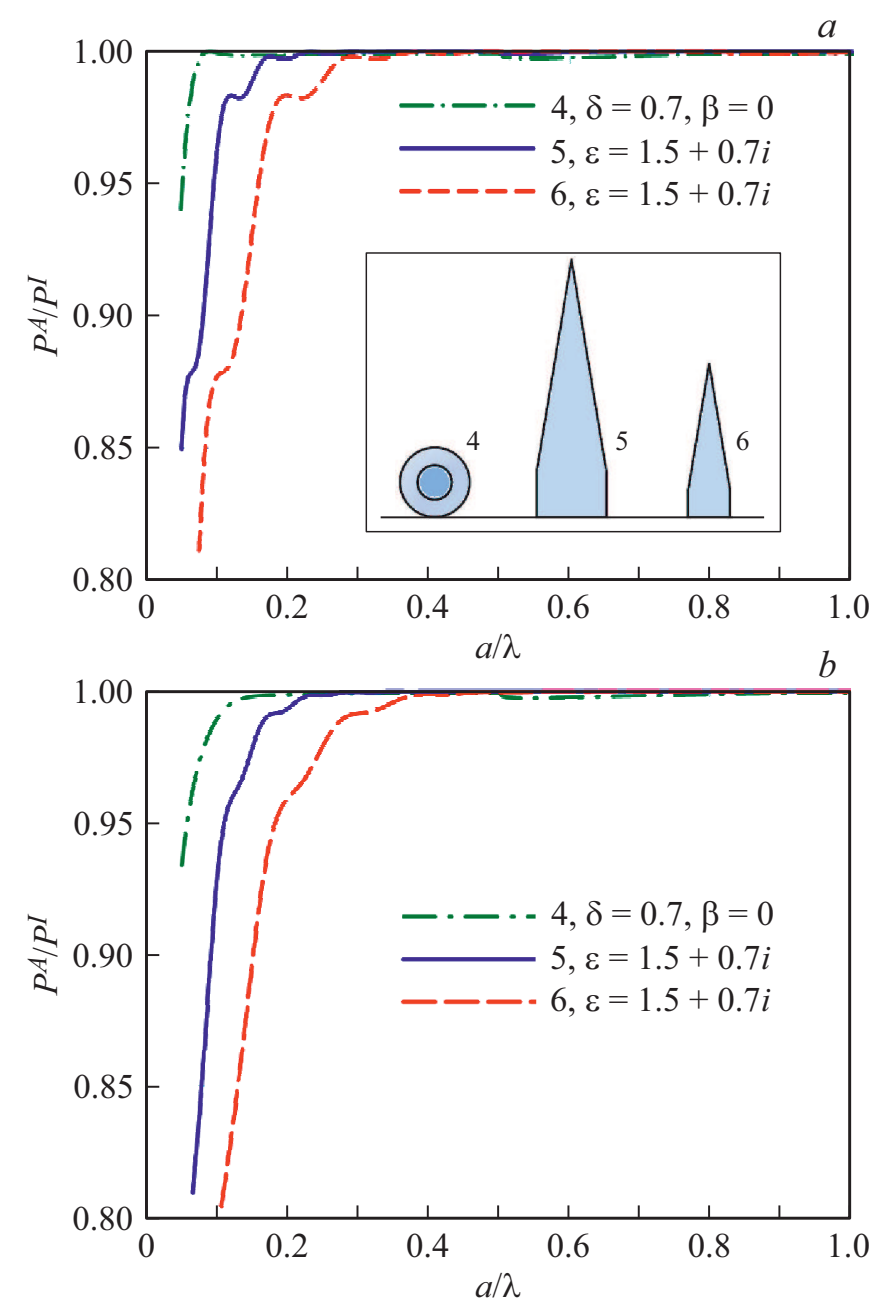

Рис. 6. Сравнение характеристик поглощения в зависимости от поперечных размеров элементов в структурах с черными дырами 4 и клиновидными элементами 5 и 6 в случаях $E$ поляризации $(a)$ и $H$-поляризации $(b)$. 

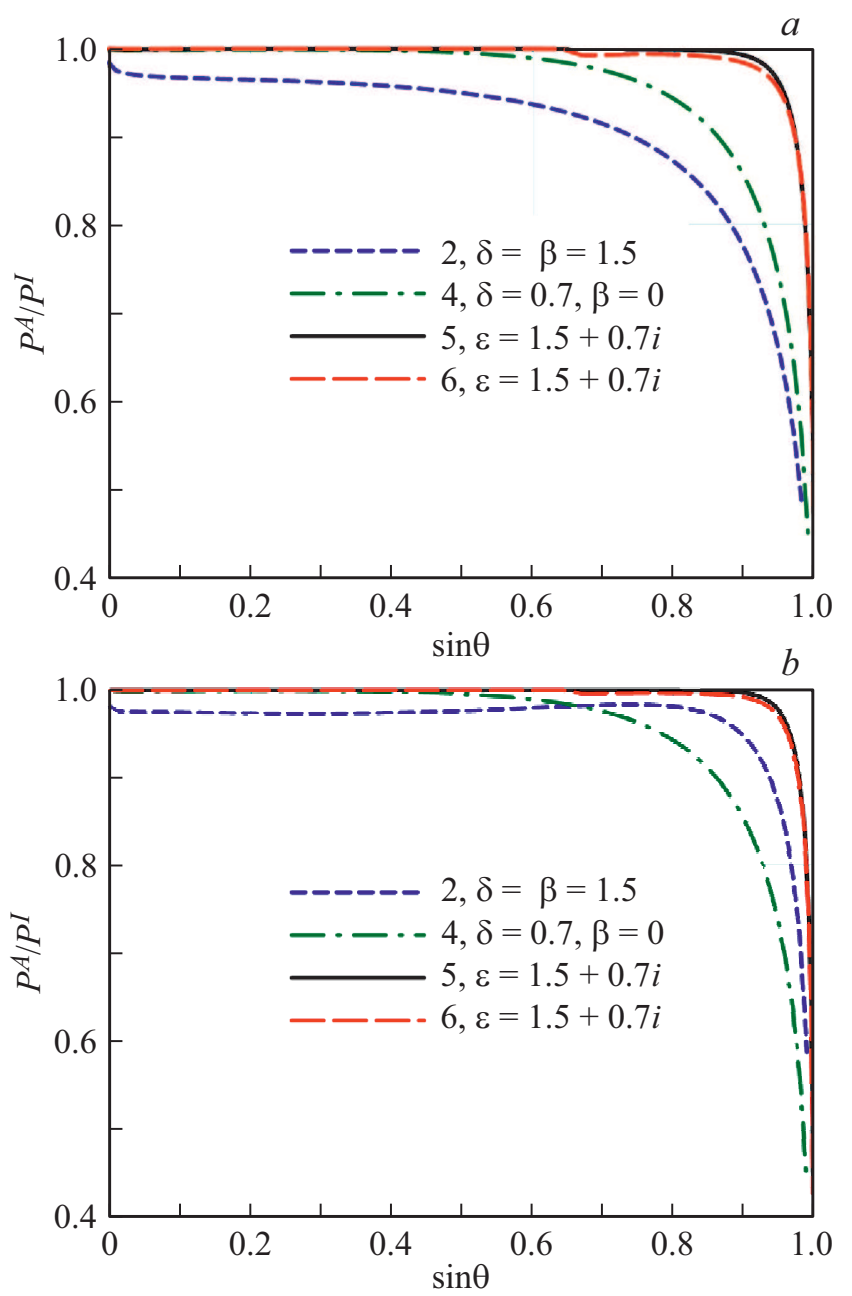

Рис. 7. Сравнение характеристик поглощения в зависимости от угла падения волны в структурах с черными дырами 2 и 4 и клиновидными элементами 5 и 6 в случаях $E$-поляризации $(a)$ и $H$-поляризации $(b)$.

Рассматривая возможность применения структур с черными дырами в качестве покрытий безэховых камер СВЧ-диапазона, представляет интерес сравнить их характеристики поглощения с характеристиками стандартных структур с пирамидальными элементами или их двумерными аналогами в виде структур с клиновидными элементами, рассмотренных в [20] и других работах, цитируемых там, расположенными на экране. Такой элемент состоит из прямоугольного пьедестала, ширину и высоту которого обозначим как $d$ и $h_{0}$ соответственно, и клиновидной части шириной $d$ у основания и высотой $h_{1}$. Указанная ширина элемента равна периоду структуры. Типичными пропорциями являются $h_{0}=0.65 d$ и $h_{1}=3 d$. Будем считать также, что диэлектрическая проницаемость элемента равна $\varepsilon=1.5+0.7 i$ [20]. Далее мы рассмотрим следующие случаи клиновидных элементов, которые обозначим как поглотители 5 и 6 :

5. Ширина поглотителя равна периоду структуры с черными дырами, т. е. $d=b=2 a$.
6. Ширина поглотителя $d=a \sqrt{\pi / 2.15}$ определяется из того условия, что площадь сечения поглотителя равна площади сечения черной дыры, т. е. $\pi a^{2}$.

Результаты сравнения поглощенной мощности в рассматриваемых структурах показаны на рис. 6 в зависимости от радиуса а, характеризующего поперечные размеры поглотителей при нормальном падении волны для обоих случаев поляризации, и на рис. 7 в зависимости от угла падения при $a=0.5 \lambda$. Вставка на рис. 6 , а также показывает сравнительные размеры элементов. Результаты на рис. 6 показывают, что черные дыры 4 обеспечивают более высокую эффективность поглощения при малых поперечных электрических размерах элементов (в низкочастотной области) по сравнению с клиновидным элементом 5 и имеют еще большее преимущество по сравнению с элементами 6. Причиной является слишком малая мнимая составляющая проницаемости клиновидных элементов, недостаточная для эффективного поглощения при малых размерах. Однако, как мы видим на рис. 7, клиновидные элементы обеспечивают более высокую эффективность поглощения при больших углах падения. Тем не менее можно считать, что черные дыры являются перспективной альтернативой стандартным структурам, так как позволяют существенно снизить толщину поглощающих стенок. Конечно, следует отметить, что рассмотренные радиально неоднородные цилиндрические поглотители являются более сложными для изготовления по сравнению со стандартными однородными клиновидными элементами. Однако аддитивные технологии типа трехмерной печати (3D-printing) [24], быстро развивающиеся в настоящее время, могут существенно упростить процесс изготовления в ближайшем будущем.

\section{Заключение}

В работе рассмотрена двумерная задача рассеяния электромагнитной плоской волны на одномерной периодической структуре, образованной из цилиндрических поглотителей типа черных дыр различных модификаций, расположенных на полубесконечной магнитнодиэлектрической подложке. Разработаны алгоритмы решения, соответствующие случаям $E$ - и $H$-поляризации и основанные на гибридном проекционном методе с учетом распределения профилей диэлектрической и магнитной пронициемостей в черных дырах четырех различных типов. Алгоритмы были обобщены также и на случай расположения черных дыр на идеально проводящем экране.

Разработанные алгоритмы были реализованы в нескольких программах в среде MATLAB. Программы были использованы для численного исследования влияния параметров поглотителей на поглощенную, отраженную и прошедшие мощности при расположении структуры в свободном пространстве при задании единичных значений относительных проницаемостей под- 
ложки, а также влияния экрана на характеристики структуры. Для каждого типа поглотителя были оптимизированы параметры с целью получения максимальной эффективности поглощения. Получены новые результаты характеристик поглощения и рассеяния структуры. Показано, что наиболее высокую эффективность поглощения обеспечивают магнитно-диэлектрические поглотители с профилем проницаемостей вида $1 / \rho^{2}$. Исследование структуры на экране показало, что экран заметно влияет на характеристики структуры только при малых значениях периода последней.

Характеристики структуры с черными дырами на экране, сравнивались с характеристиками известных структур с клиновидными поглощающими элементами с тем же периодом или с той же площадью сечения элемента, что и черные дыры. Показано, что структура с магнитно-диэлектрическими черными дырами обеспечивает более высокую эффективность поглощения в широкой полосе частот и при углах падения волны не слишком близких к углу скольжения. При этом толщина такой структуры в несколько раз меньше, чем толщина структуры с клиновидными элементами.

Полученные результаты показывают хорошие перспективы дальнейших исследований, связанных с применением сферических черных дыр в качестве элементов двумерно-периодических поглощающих структур для работы в СВЧ и оптическом диапазонах с учетом развития технологии их изготовления путем трехмерной печати.

\section{Конфликт интересов}

Авторы заявляют, что у них нет конфликта интересов.

\section{Список литературы}

[1] E.E. Narimanov, A.V. Kildishev. Appl. Phys. Lett., 95, 041106 (2009).

[2] Q. Cheng, T.J. Cui, W.X. Jiang, B.G. Cai. New J. Phys., 12, 063006 (2010).

[3] A.V. Kildishev, L.J. Prokopeva, E.E. Narimanov. Opt. Express, 18 (16), 16646 (2010).

[4] W. Lu, J.-F. Jin, Z. Lin, H. Chen. J. Appl. Phys., 108, 064517 (2010).

[5] H.-W. Wang, L.-W. Chen. J. Appl. Phys., 109, 103104 (2011).

[6] Я.И. Чижевская, О.Н. Смольникова, С.П. Скобелев. Радиотехника, 4, 23 (2018). [Ya.I. Chizhevskaya, O.N. Smolnikova, S.P. Skobelev. Radioengineering, 4, 23 (2018).] http://radiotec.ru/article/20759\#english

[7] A.S. Gutman. J. Appl. Phys., 25 (7), 855 (1954).

[8] S. Li, L. Li, Z. Lin, H.Y. Chen, J. Zi, C.T. Chan. Phys. Rev. B, 82, 054204 (2010).

[9] S.I. Maslovski, C.R. Simovski, S.A. Tretyakov. New J. Phys., 18, 013034 (2016).

[10] В.Г. Веселаго. УФН, 92 (3), 517 (1967). [V.G. Veselago. Sov. Phys. Uspekhi, 10 (4), 509 (1968).]

[11] Я.И. Чижевская, С.П. Скобелев. Опт. и спектр., 127 (6), 991 (2019). [Ya.I. Chizhevskaya, S.P. Skobelev. Opt. Spectr., 127 (6), 1085 (2019).]
[12] C.A. Valagiannopoulos, S.A. Tretyakov. IEEE Trans. Antennas Propagat., 62 (10), 5089 (2014).

[13] Y. Ra'di, V. Asadchy, S.U. Kosulnikov, M.M. Omelyanovich, D. Morits, A.V. Osipov, C.R. Simovski, S.A. Tretyakov. ACS Photonics, 2 (5), 653 (2015).

[14] P. Bradley, M. Munoz, C. Brennan, Y. Hao. Proc. 12th European Conf. on Antennas and Propagation (EuCAP'2018). (London, UK, Apr. 8-13, 2018)

[15] C. Yang, W.D. Burnside, R.C. Rudduck. IEEE Trans. on Antennas Propagat., 40 (6), 652 (1992).

[16] А.А. Щербаков, А.В. Тищенко. Квант. электрон., 40 (6), 538 (2010) [A.A. Shcherbakov, A.V. Tishchenko. Quant. Electron., 40 (6), 538 (2010).]

[17] K. Dossou, M.A. Byrne, L.C. Botten. J. Comput. Phys., 219, 120 (2006).

[18] F. Baida, A. Belkhir. ]it In Gratings: Theory and Numeric Applications, ed. E. Popov (Institut Fresnel, CNRS, AMU, 2012), p. 333. www.fresnel.fr/files/gratings/ Chapter9.pdf

[19] E. Popov. In Gratings: Theory and Numeric Applications, ed. E. Popov (Institut Fresnel, CNRS, AMU, 2012), p. 247. www.fresnel.fr/files/gratings/ Chapter7.pdf

[20] С.П. Скобелев, О.Н. Смольникова. РЭ, 57 (10), 1066 (2012). [S.P. Skobelev, O.N. Smolnikova. JCTE, 57 (10), 1073 (2012).]

[21] Gratings: Theory and Numeric Applications, ed. E. Popov (Institut Fresnel, CNRS, AMU, 2012) www.fresnel.fr/numerical-grating-book

[22] W.P. Pinello, R. Lee, A.C. Cangellaris. IEEE Trans. on Microwave Theory and Techniques, 42 (12), 2294 (1994).

[23] С.П. Скобелев, А.А. Япарова. РЭ, 52 (3), 311 (2007). [S.P. Skobelev, A.A. Yaparova. JCTE, 52 (3), 293 (2007).]

[24] Guest Editorial Special Cluster on Three-Dimensional Printed Antennas and Electromagnetic Structures, Eds. S.K. Sharma, H. Xin, B.-I. Wu, J.C. Vardaxoglou, C.H. Chan. IEEE Antennas and Wireless Propagation Letters, 17 (11), 1998 (2018). 